\title{
Les comparateurs (médicamenteux et non médicamenteux) pour l'autorisation de mise sur le marché, pour la santé publique, pour les payeurs et au niveau européen
}

\author{
Driss Berdaï ${ }^{1}$, Jean-Michel Hotton ${ }^{2}$, Philippe Lechat ${ }^{3}$ et les participants à la Table Ronde $n^{\circ} 1$ de Giens XXV $V^{\star}$ \\ 1 CHU de Bordeaux, Bordeaux, France \\ 2 Pfizer, Paris, France \\ 3 Afssaps, Saint-Denis, France
}

Texte reçu le 29 mars 2010 ; accepté le 8 juin 2010

\begin{abstract}
Mots clés :
essai clinique ; groupe contrôle ; comparateur ; bénéfice clinique ; assurance maladie ; recommandation

Résumé - La comparaison est un fondement de l'évaluation du médicament. Se pose dès lors le problème du choix du comparateur de tout nouveau traitement, ce d'autant plus qu'existent de larges disparités des pratiques médicales et des conditions d'utilisation des comparateurs, en fonction des aires géographiques et de leur évolution dans le temps. Le choix du ou des comparateurs doit répondre aux attentes parfois différentes des autorités d'enregistrement puis de prise en charge assurantielle. Le comparateur universel permettant de répondre à toutes les questions d'évaluation clinique n'existe pas. Le placebo lorsqu'il est utilisable reste une référence pour le dossier d'Autorisation de Mise sur le Marché (AMM) mais n'exclut pas l'utilisation du médicament de référence disponible sur le marché et prescrit dans des conditions d'efficacité optimales. Le traitement de référence est parfois de choix difficile dû à l'absence de recommandation thérapeutique validée ou lorsque ces recommandations sont différentes selon les territoires. Un étoffement et une harmonisation internationale des référentiels de prescription (de bon usage) renforceraient la robustesse et l'efficience des efforts de recherche clinique quant à la pertinence de l'exercice de comparaison aux traitements de référence. Ce principe est tout aussi applicable à l'utilisation d'un comparateur non médicamenteux lorsqu'il est reconnu comme le comparateur de référence dans la prise en charge de la pathologie en question. Dans la mesure du possible, la recherche de consensus doit de la même façon viser à définir dans les recommandations de développement clinique des seuils de significativité de quantité d'effet. L'optimisation des informations mises à disposition suite à essais cliniques pourra en outre être aidée par le développement de l'usage de méthodologies permettant lors d'une étude de non infériorité sur le critère principal d'évaluer une supériorité sur des critères secondaires. Enfin, le développement des consultations précoces de la Haute Autorité de Santé contribuerait à mieux adapter les essais cliniques de phase III aux questions relatives à l'évaluation de l'amélioration du service médical rendu.
\end{abstract}

\section{Introduction}

L'exercice de comparaison est à la base de la méthode d'évaluation scientifique en médecine, notamment dans le cadre des essais thérapeutiques contrôlés. ${ }^{1]}$

Cette démarche expérimentale est toutefois rendue difficile par la diversité des comparateurs médicamenteux et non médi-

\footnotetext{
* Pour la liste des participants, voir en fin d'article.
}

camenteux applicables à des situations cliniques similaires. Pourtant, cette méthode est essentielle afin d'apporter, lors du développement clinique des médicaments, des réponses pertinentes aux questions de prise en charge optimale des patients traités. Au-delà, les informations fournies par les essais cliniques doivent permettre de constituer les dossiers d'Autorisation de Mise sur le Marché $(\mathrm{AMM}),{ }^{[2]}$ de répondre aux questions relatives à leur prise en charge par la collectivité et lors de la fixation de leur prix, ${ }^{[3,4]}$ 
ces étapes réglementaires étant incontournables avant la mise à disposition des médicaments à une majorité des patients pouvant en bénéficier. ${ }^{[5]}$

\section{Les contraintes identifiées}

2.1. L'absence de consensus sur la méthode de prise en charge optimale des patients et des pathologies visées lexemple: absence de consensus de pratique médicale)

Le progrès thérapeutique repose en particulier sur les données mises à disposition suite à plusieurs milliers d'essais cliniques nouveaux initiés chaque année. ${ }^{[6]}$ Au-delà des limites à extrapoler les résultats observés en situation expérimentale à la vie réelle, ${ }^{[7]}$ une autre difficulté posée aux cliniciens consiste à traduire ces résultats en données opérationnelles applicables au quotidien. ${ }^{[8,9]}$ Cette difficulté est tempérée par le produit de l'évaluation des médicaments par les autorités compétentes. Ces dernières valident et mettent à disposition des documents opposables présentant une réelle valeur pratique (exemple : résumés des caractéristiques des produits ou RCP, avis et synthèses d'avis de la Commission de la Transparence, fiches d'information thérapeutique, etc.). Cet effort considérable d'évaluation systématique et de codification des usages a permis un important progrès au fil du temps dans l'harmonisation des pratiques.

Cependant, les pratiques professionnelles restent variables pour des conditions médicales similaires. Ces différences peuvent en particulier être expliquées par l'absence de recommandations valides de pratique médicale (référentiel de bon usage) ou par des recommandations divergentes entre pays ou entre institutions. Or, des pratiques cliniques validées et mises à jour régulièrement seraient des plus pertinentes à considérer lorsqu'il s'agit de choisir un groupe de comparaison lors d'un essai clinique d'un nouveau médicament. ${ }^{[10,11]}$ On comprend alors mieux l'importance du développement de référentiels de pratique clinique harmonisés au niveau international ou Européen au minimum afin d'optimiser les essais cliniques pivots.

\subsection{La variabilité géographique des pratiques médicales de référence}

Un important effort d'harmonisation internationale est mené depuis de nombreuses années dans le domaine de l'évaluation des médicaments, ${ }^{[12]}$ que ce soit par la mise à disposition de recommandations de développement clinique [à l'échelle européenne au travers de l'European Medicines Agency (EMA) et du Committee for Medicinal Products for Human Use (CHMP), ou internationale au travers du processus International Conference on Harmonisation (ICH)] mais aussi par la mutualisation des moyens d'évaluation des dossiers d'AMM aboutissant notamment à une homogénéité croissante des résumés des caractéristiques des produits au sein de l'Union Européenne. Cependant, seuls les médicaments les plus récents ont fait l'objet de telles procédures. En outre, en pratique clinique, les recommandations de développement clinique ainsi que les recommandations de prise en charge thérapeutique sont établies par pathologies et non par spécialités pharmaceutiques particulières. Il en résulte une disparité toujours excessive d'usages cliniques des médicaments selon les territoires, sans pour autant pouvoir être expliquée par des raisons médicalement valides (exemple : profils génétiques différents nécessitant un mode d'administration adapté). Au-delà, certains référentiels recommandent même des pratiques thérapeutiques différentes selon les territoires bien que les pathologies visées soient d'expression clinique similaire (exemple : asthme).

\subsection{Les variations de conditions d'utilisation des traitements de référence selon les territoires (formulations, posologies... )}

Au-delà des considérations développées dans le paragraphe précédent, le choix d'un comparateur unique lors d'un essai clinique pivot de phase III peut être compliqué par des modes d'utilisation différents d'un même principe actif selon les territoires considérés tels que : formes pharmaceutiques (solution, forme solide retard, etc.), posologies, chronologies des prises ou voies d'administration. Ceci est notamment le cas de comparateurs dont les AMM ont pu être différentes d'un pays à l'autre au sein de l'Union Européenne avant application de la procédure centralisée.

2.4. L'évolution des pratiques dans le temps (exemple du médicament de référence dont l'AMM est octroyée sur la période de développement clinique d'un médicament visant la même indication)

Dans les domaines à forte activité de recherche clinique, le rythme d'innovation peut être tel que la référence thérapeutique peut changer sur la période de temps d'un développement clinique. De la sorte, un comparateur valide au moment de l'élaboration d'un protocole peut devenir obsolète lors de la soumission d'un dossier de demande d'autorisation de mise sur le marché ou, a fortiori, lors de la demande de prise en charge par les organismes d'assurance maladie. 


\subsection{Des informations multiples à apporter à de nombreux acteurs et autorités internationales sur la base de données provenant d'un seul développement clinique et d'un nombre limité d'études de phase III}

Les informations attendues à l'issue des essais cliniques des médicaments sont multiples. ${ }^{[13]}$ Selon les questions posées, les choix méthodologiques et de constitution des groupes comparateurs varient, ${ }^{[14]}$ en particulier :

- L'usage du placebo ${ }^{[15]}$ (dans les cas où celui-ci est utilisable) est habituellement requis par les Autorités compétentes en vue de constituer un dossier de demande d'autorisation de mise sur le marché;

- L'utilisation d'un traitement de référence (médicamenteux ou non) est nécessaire à l'évaluation du bénéfice et des risques relatifs liés à la nouvelle thérapeutique évaluée et à ce titre sera fortement recommandée pour un dossier de demande d'AMM et à l'appui d'une demande de prise en charge par les organismes d'assurance maladie; ;16]

- D'autres méthodologies ou analyses spécifiques peuvent être nécessaires afin de répondre à des besoins d'évaluation d'efficience (cost-effectiveness) ou encore d'impact budgétaire (budget impact analysis).

La déclinaison de l'ensemble de ces besoins d'évaluation avec les nécessaires adaptations aux contextes locaux conduit à un nombre d'essais cliniques souvent important. A contrario, les impératifs de rigueur méthodologique inviteraient plutôt à inclure un maximum de patients dans un minimum d'essais cliniques afin d'en maximiser la puissance et plus généralement l'efficience.

\section{Propositions de recommandations}

\section{1. La comparaison reste le fondement de toute évaluation}

Des essais cliniques contre placebo et/ou comparateurs actifs sont nécessaires sauf exceptions dans certaines pathologies rares. Dans le cas de ces dernières, des comparaisons historiques sont possibles si elles sont justifiées et suffisamment bien référencées. L'absence d'un groupe contrôle peut être acceptée si la méthodologie permet de démontrer un effet clinique majeur attendu et argumenté (notamment en terme de survie ou sur d'autres critères d'efficacité objectifs et cliniquement pertinents tels que la survie sans récidive ou la guérison).
3.2. L'utilisation d'un comparateur non médicamenteux peut, voire doit, être appliquée dès lors qu'un tel comparateur existe et qu'il est reconnu comme étant de référence par les recommandations pertinentes (guidelines)

Ce type de comparaison ne peut dispenser d'une évaluation versus placebo du médicament à l'étude (sauf limite éthique). Lors de l'utilisation d'un comparateur non médicamenteux, la robustesse des résultats peut être affectée par la difficulté à réaliser dans de bonnes conditions une étude en double aveugle. Par ailleurs, les infrastructures nécessaires à l'administration des deux types de traitement peuvent largement différer et entraîner des problèmes de transposabilité des résultats d'une étude à la population générale. Néanmoins, ce type de comparaison peut avoir un intérêt en termes d'élaboration de stratégies thérapeutiques ou pour soutenir des décisions de santé publique (exemple : appendicectomie versus antibiothérapie). ${ }^{[17]}$

\subsection{Le comparateur idéal permettant de répondre à l'ensemble des questions d'évaluation clinique n'existe pas}

Son choix sera donc déterminé au mieux par les objectifs visés (pharmacologique, thérapeutique, économique, politique de santé) mais aussi par le temps de l'évaluation (pré- ou postAMM), le contexte, notamment médical ou culturel, la méthode de comparaison, les conditions d'utilisation du comparateur, le type de bénéfice étudié ou le contexte épidémiologique (âge des populations, pathologies chroniques).

\subsection{Pour I'AMM, la comparaison au placebo reste indispensable (lorsqu'elle est possible sans perte de chance pour le patient)}

D'autre part, l'utilisation d'un comparateur actif de référence est d'autant plus souhaitable que la classe thérapeutique d'appartenance du médicament évalué est étoffée. Le besoin d'étude versus comparateur actif est proportionnel à l' ancienneté de la classe, et, dans une classe avec plusieurs représentants, un nouvel entrant devrait idéalement être comparé au produit antérieur le plus performant dans l'indication visée. Dans le cadre de l'AMM, la comparaison à des produits possédant le même mécanisme d'action sera recherchée. 


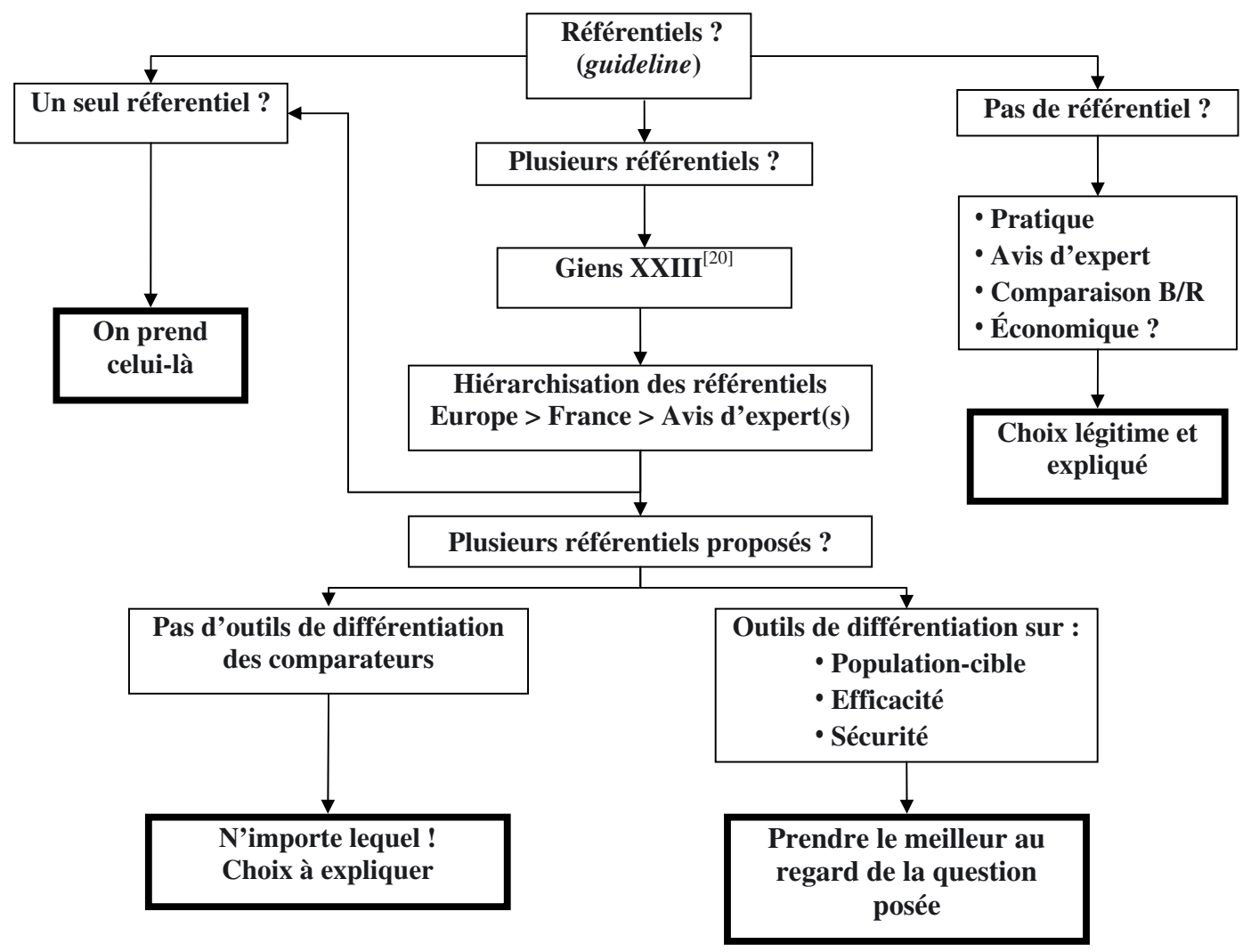

Fig. 1. Utilisation des référentiels dans le choix des comparateurs. $\mathbf{B} / \mathbf{R}$ : rapport bénéfice/risque.

\subsection{En matière d'évaluation de l'Amélioration du Service Médical Rendu (ASMR), la quantification de l'effet ne peut se faire que par comparaison au traitement médicamenteux de référence du moment, ou à défaut par rapport à la stratégie thérapeutique courante}

Le comparateur est à choisir en fonction des référentiels de pratique (cf. infra), ce qui met en exergue l'importance d'une harmonisation internationale, ou européenne a minima, des recommandations de pratique professionnelle. Les conditions d'utilisation (posologie, durée...) du comparateur doivent être celles autorisant l'expression maximale de son efficacité dans le cadre du référentiel (AMM, autres...).

\subsection{L'utilisation des référentiels est indispensable dans le choix du comparateur}

Les choix en fonction des référentiels existants sont décrits dans la figure 1 .

\subsection{La mise en œuvre de méthodologies permettant de montrer une supériorité sur des critères secondaires dans une étude de non infériorité sur le critère principal est souhaitable}

On peut en effet observer, dans une étude comparant deux produits actifs, une non-infériorité en termes d'efficacité sur le critère principal et une supériorité sur d'autres critères mesurés (exemple : tolérance clinique, qualité de vie). L'utilisation de ce type de méthodologie impose que le (ou les) critère(s) secondaire(s) sur le(s)quel(s) va porter l'analyse soient clairement définis au préalable dans le protocole, et ne puissent pas être choisis à posteriori en fonction des résultats observés.

3.8. Le développement au niveau de la Haute Autorité de Santé (HAS) de consultations précoces en fin de phase II, à l'instar des avis scientifiques délivrés par I'EMA et l'Agence Française de Sécurité Sanitaire des Produits de Santé (Afssaps), offrirait aux industriels du médicament l'opportunité de mieux adapter les plans de développement de phase III

Outre une présentation du plan de développement envisagé, pourraient alors être discutés le choix du ou des comparateurs, les 


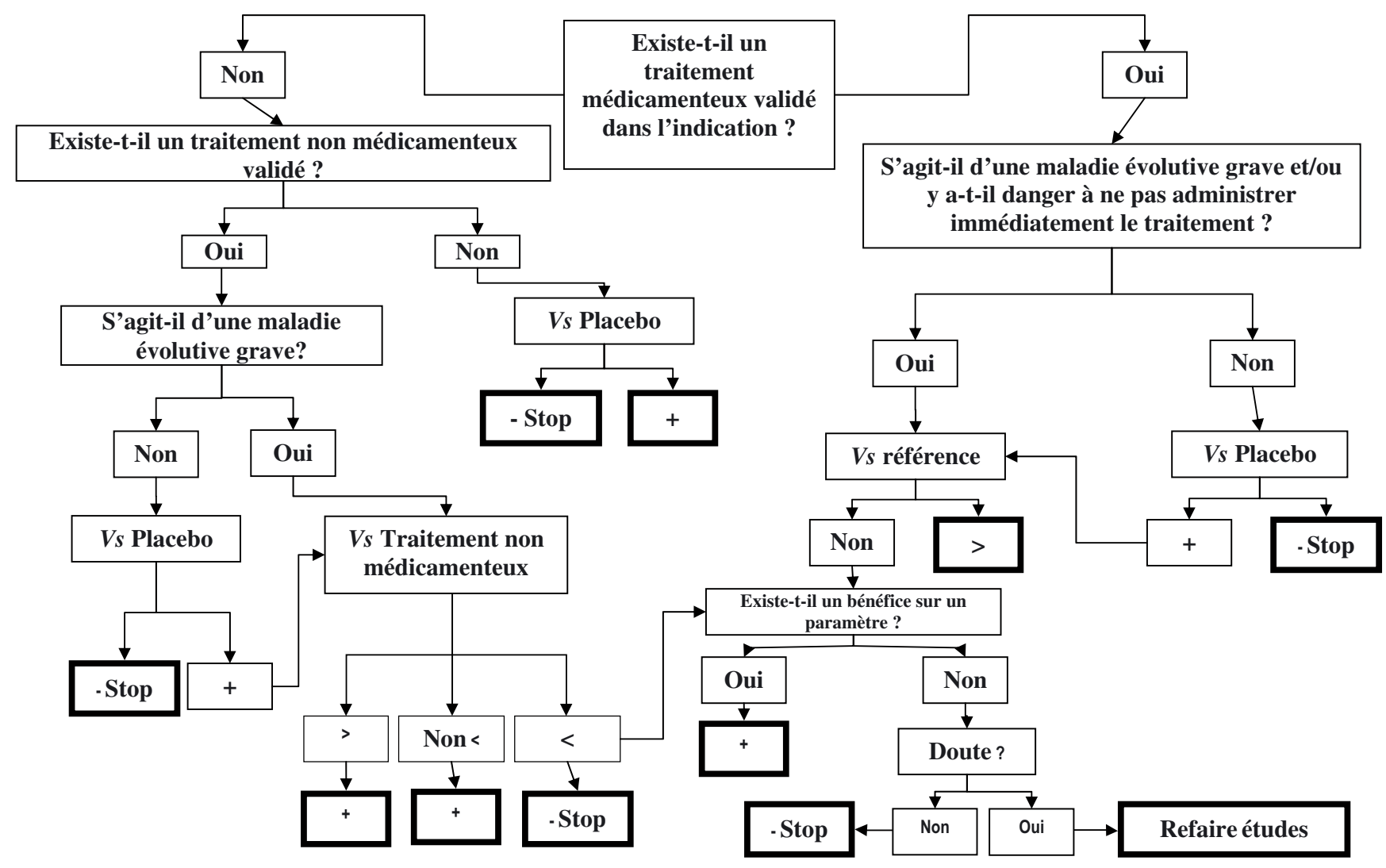

Fig. 2. Utilisation des comparateurs dans le cadre du développement clinique d'un médicament.

modalités d'administration (posologies, rythme d'augmentation des doses,... ), la quantité d'effet attendue, les populations visées, les conditions de transposabilité des études,... La mise en place de telles consultations précoces (sans engagement des Autorités en fonction des résultats d'essais) apparaît d'autant plus nécessaire qu'elles sont déjà une pratique courante de plusieurs agences de Health Technology Assessment, et notamment du National Institute for Health and Clinical Excellence (NICE) ${ }^{[18]}$ et du Canadian Agency for Drugs and Technology in Health (CADTH). ${ }^{[19]}$

\subsection{Réfléchir, à un niveau national et/ou européen, au choix du comparateur dans l'analyse des relations précises entre quantité d'effet observée et bénéfice clinique attendu}

La rédaction de recommandations internationales applicables aux développements cliniques, notamment au travers du processus $\mathrm{ICH}$, ou encore au niveau Européen [European Medicines Agency Efficacy Working Party (EMA EWP) guidelines], a contribué à harmoniser la méthodologie de développement clinique des médicaments et à augmenter la robustesse des résultats observés. Dans la continuité de cet effort et afin de renforcer la pertinence de ces résultats et l'efficience des moyens mobilisés à cette fin, il serait souhaitable d'enrichir ces recommandations d'informations relatives au choix des comparateurs et aux quantités minimales d'effets permettant d'affirmer un bénéfice clinique.

\section{Conclusion}

Lors de l'évaluation des médicaments, le choix des comparateurs reste un problème délicat dans la mesure où, en règle générale, les données comparatives issues des études de phase III ne permettent pas toujours de répondre à de multiples questions. Dans ce cadre, la comparaison au placebo reste un passage obligé (sauf justification appropriée). La comparaison directe avec un produit actif, s'il existe, reste la seule méthode robuste pour démontrer une amélioration du service médical rendu. Le choix des comparateurs actifs, qu'ils soient médicamenteux ou non médicamenteux, repose sur une utilisation soigneuse des référentiels. La démarche de développement et le choix des comparateurs sont ainsi décrits dans la figure 2. L'utilisation de méthodologies statistiques appropriées associant la démonstration d'une non-infériorité sur un critère (par exemple l'efficacité) et la supériorité sur un autre (par exemple la tolérance ou la qualité de vie) permettrait de tirer davantage d'enseignements des études 
comparatives ainsi menées. Enfin, des échanges accrus avec la Haute Autorité de Santé (HAS) en fin de phase II permettraient de mieux anticiper les attentes des Autorités nationales et, de fait, d'améliorer les plans de développement de phase III.

Participants. Alexandre Barna (Direction Générale de la Santé, Paris), Laurent Becquemont (AP-HP, Paris), JeanFrançois Bergman (AP-HP, Paris), Corine Bernaud (Laboratoire Astra-Zeneca), Isabelle Boucot (Laboratoire GlaxoSmithKline), Isabelle Cheiney (Direction de la Sécurité Sociale, Paris), Michel Cucherat (Hôpital Laënnec, Lyon), Anne d'Andon (Haute Autorité de Santé, Saint-Denis la Plaine), Jérôme d'Enfert (Laboratoire Roche), Alain Francillon (Laboratoire Novartis), Elisabeth Frauger (CIC, UPCET, AP-HM, Marseille), Danièle Girault (Laboratoire Wyeth), Jean-Marc Harlin (Mutualité Sociale Agricole), Alain Joseph (Laboratoire Pierre Fabre), Catherine Lassale (LEEM-ARIIS), Florence Mahlberg-Gaudin (Laboratoire Servier), Thierry Moreau-Defarges (Cyclamed, Boulogne), Mira Pavlovic (Afssaps, Saint-Denis), Sophie Ravoire (Laboratoire Lilly), Lise Rochaix (Haute Autorité de Santé, Paris), Eric Vicaut (AP-HP, Paris).

\section{Références}

1. EU standard of medicinal product registration: clinical evaluation of risk/benefit - The role of comparator studies. Ref. EMEA/119319/04, October 2004 http: //www . ema. europa. eu

2. Van Luijn JCF, Gribnau FWJ, Leufkens HGM. Availability of comparative trials for the assessment of new medicines in the European Union at the moment of market authorization. Br J Clin Pharmacol 2006; 63: 159-62

3. Chalkidou K, Tunis S, Lopert R, et al. Comparative effectiveness research and evidence-based health policy: experience from four countries. The Milbank Quarterly 2009; 87: 339-67

4. Clement FM, Harris A, Li JJ, et al. Using effectiveness and cost-effectiveness to make drug coverage decisions: a comparison of Britain, Australia, and Canada. JAMA 2009; 302: 1437-43
5. Marley J. Efficacy, effectiveness, efficiency. Aust Prescr 2000; 23: 114-5

6. Chalmers I, Glasziou P. Avoidable waste in the production and reporting of research evidence. Lancet 2009; 374: 86-9

7. Atkins D. Creating and synthesizing evidence with decision makers in mind. Med Care 2007; 45: S16-S22

8. Mitka M. Studies comparing treatments ramp up. JAMA 2009; 301: 1975

9. Berwick DM. The science of improvement. JAMA 2008; 299: 1182-4

10. Dawson L, Zarin DA, Emanuel EJ, et al. Considering usual medical care in clinical trial design. PLoS Med 2009; 6: 1-6

11. Rawlins M. Harveian Oration - De testimonio: on the evidence for decisions about the use of therapeutic interventions. Lancet 2008; 372: 2152-61

12. ICH topic E10. Choice of control group in clinical trials. Ref. CPMP/ICH/364/96, January 2001 http : //www . ema . europa . eu

13. Glickman SW, McHutchinson JG, Peterson ED, et al. Ethical and scientific implications of the globalisation of clinical research. N Eng J Med 2009; 360: $816-23$

14. Luce BR, Kramer JM, Goodman SN, et al. Rethinking randomized clinical trials for comparative effectiveness research: the need for transformational change. Ann Intern Med 2009; 151: 206-9

15. EMEA/CPMP position statement on the use of placebo in clinical trials with regard to the revised declaration of Helsinki. Ref. EMEA/17424/01, June 2001 http: //www . ema. europa. eu

16. Garattini S, Chalmers I. Patients and the public deserve big changes in evaluation of drugs. BMJ 2009; 338: 804-6

17. Andersson RE, Petzold MG. Nonsurgical treatment of appendiceal abscess or phlegmon: a systematic review and meta-analysis. Ann Surg 2007; 246: 741-8

18. http://www.nice.org.uk/aboutnice/scientificadvice/ AboutScientificAdvice.jsp

19. http://www.cadth.ca/index.php/en/cdr/filing-submission/ pre-submission-meetings

20. Table Ronde $n^{\circ} 2$ de Giens XXIII. Le bon usage du médicament : définition, référentiels, périmètre et champ d'application. Therapie 2008; 63: 267-73

Correspondance et offprints : Driss Berdaï, Service de Pharmacologie Clinique, CHU de Bordeaux, Zone Nord Carreire, Bâtiment 1A, Place Amélie-Raba-Léon, 33076 Bordeaux Cedex, France.

E-mail : driss.berdai@u-bordeaux2.fr 